\title{
Performance Anomaly of the IEEE 802.11 DCF in Different Frame Error Rate Conditions
}

\author{
Koohong Kang*
}

\begin{abstract}
We propose an analytic model to compute the station's saturated throughput and packet delay performance of the IEEE 802.11 DCF (Distributed Coordination Function) in which frame transmission error rates in the channel are different from each other. Our analytic model shows that a station experiencing worse frame error rates than the others suffers severe performance degradation below its deserved throughput and delay performance. 802.11 DCF adopts an exponential back-off scheme. When some stations suffer from high frame error rates, their back-off stages should be increased so that others get the benefit from the smaller collision probabilities. This impact is then recursively applied to degrade the performance of the victim stations. In particular, we show that the performance is considerably degraded even if the frame error rate of the victim station satisfies the receiver input level sensitivity that has been specified in the IEEE 802.11 standard. We also verify the analytic results by the OPNET simulations.
\end{abstract}

Keywords_IEEE 802.11 DCF, Wireless LAN, Throughput and Delay Performance

\section{INTRODUCTION}

In recent the Smart Era, IEEE 802.11 Wireless Local Area Network (WLAN) has emerged as a promising technology for wireless access by smart mobile devices. Today most installed WLANs utilize the infrastructure mode that requires the use of one or more access points (APs), even if an optional ad-hoc mode allows users to spontaneously form a WLAN without APs for public-safety and military applications [1,2]. The IEEE 802.11 standard defines the specification for the two types access methods: the Distributed Coordination Function (DCF), which uses CSMA/CA to allow for contended access to the wireless media; and the Point Coordination Function (PCF), which provides collision-free access via arbitration by a Point Coordinator that resides in the AP. DCF uses two access mechanisms for packet transmission: the basic access or the optional Request-To-Send/Clear-To-Send (RTS/CTS) mechanism. However, the PCF is very rarely implemented in currently available devices, and the RTS/CTS option is rarely turned on in the practical infrastructure IEEE 802.11 WLANs due to high overhead. In this paper we limit our investigation to the basic DCF scheme in the infrastructure WLAN.

A lot of works on the issues of fairness and performance anomaly of the IEEE 802.11

\footnotetext{
※ I would like to thank Dr. D. Malone with the Hamilton Institute in National University of Ireland for the many valuable discussions regarding the draft version of this paper. I would also like to thank Dr. H. Choi with the Sprint Applied Research and Advanced Technology Labs in California for her valuable discussions on the final version of this paper Manuscript received February 10, 2012; accepted May 25, 2012.

Corresponding Author: Koohong Kang

* Dept. of Information and Communication Engineering, Seowon University, Cheongju, Korea (khkang@seowon.ac.kr)
} 
WLANs have been discussed [3,4]. These works are becoming more important because the radio channels should be shared among the users fairly and efficiently in present-day overcrowded Wi-Fi hotspots. However, to the best of our knowledge, all of them consider the performance variations due to the differences in the transmission rates among wireless stations using link rate adaptation schemes such as an automatic rate fallback (ARF).

Recently, as the price of APs has been getting cheaper, we deploy them very densely in public or private places. Therefore, the deployed scope of an infrastructure WLAN is so small that every station has a strong signal quality to operate with its full speed. In this case, the ARF responds to frame collisions that cannot be distinguished from frame transmission errors, and then it significantly decreases throughput when transmission failures are caused by collisions [4]. That is, we prefer to use a fixed transmission rate in this case.

In this paper, we focus on the performance anomaly of the IEEE 802.11 DCF where all of the stations are fixed at the same transmission rate but have different frame error rates (FERs). We show that the impact of this anomaly is totally different from the known results of multi-rate IEEE 802.11 WLANs because the host causing this problem does not penalize other hosts apart from itself. For this purpose, we present an analytic model by extending the throughput analysis introduced by Bianchi [6]. In particular, we show that the performance is considerably degraded even if the frame error rate of the victim station satisfies the receiver input level sensitivity that has been specified in the IEEE 802.11 standard. The rest of this paper is organized as follows: Section 2 presentsan analytic model to compute the station's saturated throughput and packet delay performance of the IEEE 802.11 DCF in which the frame transmission error rates in the channel are different from each other. Section 3 provides several numerical results to show the performance anomaly. Section 4 validates the analytic model through simulation, and finally Section 5 concludes the paper.

\section{Performance of IEEE 802.11 DCF in Different Frame ErRor RATE CONDITIONS}

Bianchi [6] presents an analytic model to compute the 802.11 DCF throughput based on a discrete Markov chain under the assumption of saturated traffic condition. Later Malone et al. [7] extended this model for the non-saturated and heterogeneous traffic conditions, and $\mathrm{Ni}$ et al. [8] simply used it to study the impact of frame transmission error on the throughput, and Chatzimisios et al. [9] presented a packet delay analysis for theIEEE 802.1b protocol by extending the Bianchi's model. In this section, for the sake of clarity, we use the same Markov chain model for back-off window size in error-prone networks using the same symbol notations that appeared in [8]. We also extend the equations obtained in [7-9] for our specific purposes.

\subsection{Throughput Analysis}

Suppose there are $M$ groups of stations labeled $i=1,2, \ldots, M$. We assume that for all of the groups, stations have the same station parameters including the 802.11 MAC and PHY layer parameters, while they have different frame transmission error rates in each group. Let $s(t)$ and $b(t)$ be the stochastic processes representing the back-off stage and the back-off counter respectively for a given station (STA) at time $t$. To model the bi-dimensional process $\{b(t), s(t)\}$ of the random back-off behavior of any given STA in group $i$, we use the same discrete-time Markov 


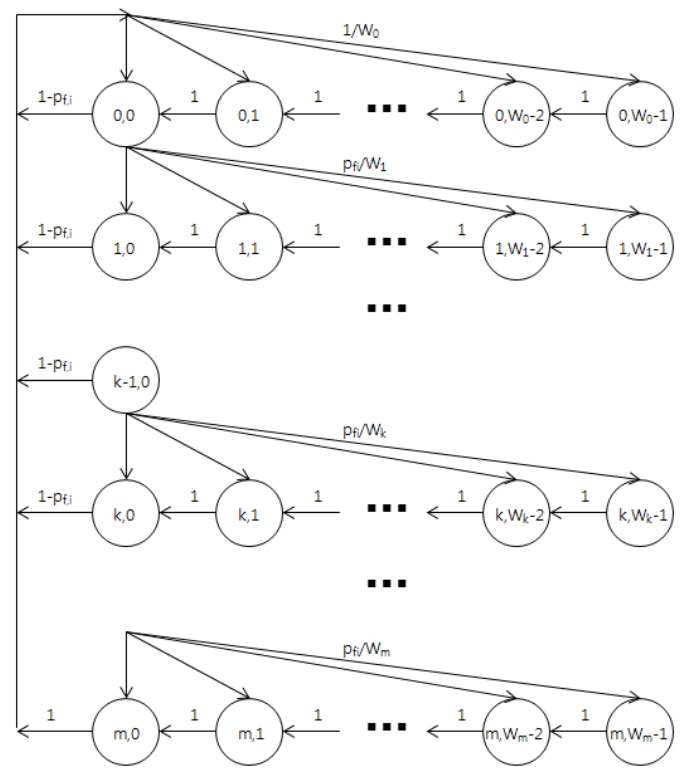

Fig. 1. Markov chain model for back-off window size

chain in [8] as shown in Fig. 1, where $p_{f, i}$ is the probability that a transmitted frame in group $i$ fails to transmit due to collision or to medium error. When an unsuccessful transmission occurs at back-off stage $k$-1, the back-off stage increases, and the new initial back-off value is uniformly chosen in the range of $\left[0, W_{k}-1\right] . W_{k}$ is the current contention window (CW) size and $k \in[0, m] . m$ denotes the retry limit number of any transmitted frame and $W_{k}=2^{k} \cdot W$, where $W$ is the minimum CW size. When the back-off stage reaches the retry limit and the last transmission is unsuccessful, the packet is discarded. Using the Markov chain for a given station in group $i$, the probability $\tau_{i}$ that a station in group $i$ transmits in a randomly chosen slot time is represented as follows:

$$
\tau_{i}=\frac{2\left(1-2 p_{f, i}\right)\left(1-p_{f, i}^{m+1}\right)}{\left(1-p_{f, i}\right) W\left(1-\left(2 p_{f, i}\right)^{m+1}\right)+\left(1-2 p_{f, i}\right)\left(1-p_{f, i}^{m+1}\right)}
$$

If $m$ is greater than $m$ ' which denotes the number of doubling the CW size of the exponential back-off scheme, the denominator of equation (1) should be modified as explained in [8]. The probability $p_{f, i}$ becomes:

$$
p_{f, i}=1-\left(1-\tau_{i}\right)^{n_{i}-1}\left(1-p_{e, i}\right) \prod_{\ell=1, \ell \neq i}^{M}\left(1-\tau_{\ell}\right)^{n_{\ell}}
$$

when each group has $n_{i}$ stations, where $p_{e, i}$ is the FER of a MAC data frame or the corresponding ACK frame in group $i$. Assuming these transmission errors are independent, $p_{e, i}=p_{e, i}^{\text {data }}+$ $p_{e, i}^{a c k}-p_{e, i}^{d a t a} p_{e, i}^{a c k}$, where $p_{e, i}^{d a t a}$ and $p_{e, i}^{a c k}$ are FERs of data and ACK frames respectively. When we also assume that the bit error rate $p_{b, i}$ on the up and down links on the medium are the same and that they are uniformly distributed over the whole frame, $p_{e, i}^{\text {data }}=1-\left(1-p_{b, i}\right)^{L_{d a t a}}$ 
and $p_{e, i}^{a c k}=1-\left(1-p_{b, i}\right)^{L_{a c k}}$, where $L_{\text {data }}$ and $L_{a c k}$ are the length of the data and the ACK frames. Now equation (1) and equation (2) provide $2 M$ non-linear equations which can be solved numerically for $p_{f, i}$ and $\tau_{i}$.

We are now able to express $S_{i}$ for the normalized throughput for each group $i$ as $S_{i}=$ $P_{s, i} E[P] / E_{s, i}$, where $P_{s, i}$ is the probability that a station in group $i$ successfully transmits as follows:

$$
P_{s, i}=n_{i} \tau_{i}\left(1-\tau_{i}\right)^{n_{i}-1}\left(1-p_{e, i}^{d a t a}\right)\left(1-p_{e, i}^{a c k}\right) \prod_{\ell=1, \ell \neq i}^{M}\left(1-\tau_{\ell}\right)^{n_{\ell}}
$$

and $E[P]$ is the expected time spent transmitting payload data, and $E_{s, i}$ is the average length of a slot time. For the ease of discussion about the performance anomaly of the IEEE 802.11 DCF in different FER conditions, we will just consider two groups of stations $(M=2)$ from now on. Let $T_{s}$ be the time for a successful transmission, $T_{c}$ be the time for a collision, and $T_{e}^{\text {data }}$ and $T_{e}^{\text {ack }}$ be the times for transmission failures due to data and ACK frame errors, respectively. Additionally $P_{I}, P_{c, i}, P_{e, i}^{\text {data }}$, and $P_{e, i}^{a c k}$ refer respectively to the probability that a time slot on the channel is occupied because of empty, collisions, data frame errors and ACK frame errors. Thus, $E_{s, i}$ becomes

$$
E_{s, i}=\sigma P_{I}+T_{S} P_{s, i}+T_{c} P_{c, i}+T_{e}^{\text {data }} P_{e, i}^{\text {data }}+T_{e}^{\text {ack }} P_{e, i}^{a c k}
$$

where $\sigma$ is the duration of an empty slot time, $P_{I}=\left(1-\tau_{1}\right)^{n_{1}}\left(1-\tau_{2}\right)^{n_{2}}, T_{s}=2 T_{\text {PHYhdr }}+$ $T_{\text {data }}+2 \delta+T_{\text {SIFS }}+T_{a c k}+T_{D I F S}$, and for brevity $T_{s}=T_{c}=T_{e}^{\text {data }}=T_{e}^{\text {ack }}$ in the case of FHSS 1 Mbps data rate. For the first group $i=1, P_{c, 1}=1-\left(1-\tau_{1}\right)^{n_{1}}\left(1-\tau_{2}\right)^{n_{2}}-n_{1} \tau_{1}(1-$ $\left.\tau_{1}\right)^{n_{1}-1}\left(1-\tau_{2}\right)^{n_{2}} \quad, \quad P_{e, 1}^{\text {data }}=n_{1} \tau_{1}\left(1-\tau_{1}\right)^{n_{1}-1}\left(1-\tau_{2}\right)^{n_{2}} p_{e, 1}^{\text {data }} \quad$, and $\quad P_{e, 1}^{\text {ack }}=n_{1} \tau_{1}(1-$ $\left.\tau_{1}\right)^{n_{1}-1}\left(1-\tau_{2}\right)^{n_{2}}\left(1-p_{e, 1}^{\text {data }}\right) P_{e, 1}^{a c k}$. We can also obtain these equations for the second group $i=2$.

\subsection{Delay Analysis}

We compute the average delay $E_{i}[D]$ for a successfully transmitted packet in group $i$. Packet delay is defined to be the time interval from the time a packet is at the head of its MAC queue ready for transmission, until its successful reception at the intended destination. $E_{i}[D]$ is given by $E_{i}[D]=E_{i}[X] E_{s, i}$, where $E_{i}[X]$ is the average number of slot times for a successful packet transmission. $E_{i}[X]$ can be found as follows:

$$
E_{i}[X]= \begin{cases}\sum_{k=0}^{m-1} d_{k} q_{k}+d_{m} q_{m}\left(1-p_{f, i}\right), & m>m^{\prime} \\ \sum_{k=0}^{m^{\prime}-1} d_{k} q_{k}+d_{m^{\prime}}\left(\sum_{k=m^{\prime}}^{m^{\prime}-1} q_{k}+q_{m}\left(1-p_{f, i}\right)\right), & m \leq m^{\prime}\end{cases}
$$

where $d_{k}$ is the mean number of slot times of the back-off stage $k$, and $q_{k}$ is the probability of the packet to reach the back-off stage $k$; that is, $d_{k}=\left(W_{i}-1\right) / 2$ and $q_{k}=p_{f, i}^{k}, k=0,1, \cdots, m$.

\section{Numerical Results}

We first consider one group of homogeneous $N$ stations whose BERs $p_{b}$ are the same. The 
Table 1. FHSS system parameters and additional parameters used to obtain numerical results

\begin{tabular}{c|c}
\hline parameters & values \\
\hline Packet payload $(E[P])$ & 8184 bits \\
\hline MAC header $\left(M A C_{h}\right)$ & 272 bits \\
\hline PHY header $\left(P H Y_{h}\right)$ & 128 bits \\
\hline ACK frame $\left(L_{a c k}\right)$ & 112 bits \\
\hline Channel Bit Rate & 1 Mbps \\
\hline Propagation Delay $(\delta)$ & 50 us \\
\hline Slot Time $(\sigma)$ & 28 us \\
\hline$T_{\text {SIFS }}$ & 128 us \\
\hline$T_{D I F S}$ & 32 us \\
\hline Min. Window Size $(W)$ & 5 \\
\hline Retry limit number $(m)$ & 6 \\
\hline
\end{tabular}

station parameters and additional parameters that were used to obtain numerical results are shown in Table 1, where the system values are those that were specified for the FHSS (Frequency Hopping Spread Spectrum) PHY layer [5]. The station throughputs in Fig. 2(a) decline slightly up to $p_{b}=10^{-5}\left(p_{e}=0.08211\right)$, whilst they decline sharply after the threshold. The station throughputs at $p_{b}=10^{-5}$ drop by about $8.5 \%$ and $6.7 \%$ from the ones when $p_{b}=10^{-8}$ for $N=2$ and $N=11$, respectively. The performance degradation will be mitigated as $N$ is increased. For example the throughput drops by $6.26 \%$ when $N=21$. In [5], the FERs shall be specified for every 802.11 PHY mode to determine the receiver minimum input level sensitivity for the operation of a given modulation scheme. For example, the FERs of the FHSS 1 Mbps mode (802.11b) and the ERP-OFDM mode (802.11g) shall be less than $3 \%$ and $10 \%$, respectively. Hence, in this study we use $p_{b}=10^{-5}$ as the threshold of a BER upper limit even if we can show some inflated numerical results for unrealistic high BERs in WLANs. Fig. 2 (b) shows the average packet delay for $N=2,11$, and 21. As expected from the previous discussion about throughput variations, the delay also increases sharply after the BER threshold (see Fig. 2 (b)). From this first scenario,

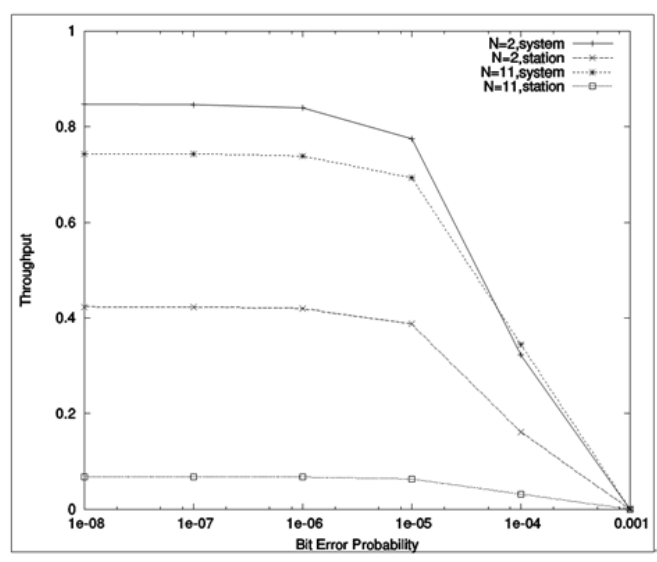

(a) System and Station Throughput

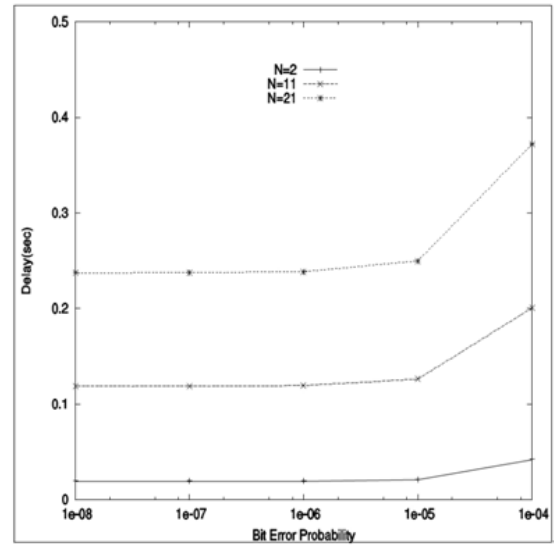

(b) Packet Delay

Fig. 2. Throughput and packet delay versus $\mathrm{p}_{\mathrm{b}}$ for $\mathrm{N}=2$ and 11 
the throughput drops maximally by $8.5 \%$ and the delay increases up to a maximum of $9.2 \%$ when $N=2$, respectively. These performance variations will be used as the references for the comparison with the different BER conditions.

Now, we consider the second scenario in which a WLAN segment services two groups of stations. We assume that there are $n_{1}$ stations in group 1 whose $p_{b, 1}$ is fixed, and there is only one station (STA2) in group 2 whose $p_{b, 2}$ varies between $10^{-8}\left(p_{e, 2}=0.000085\right)$ and $10^{-4}\left(p_{e, 2}=0.57549\right)$ depending on its location of the STA2 from the AP. Fig. 3 (or Fig. 4) shows the station throughputs (or packet delay) of STA1 (a stationin group 1) and STA2 as $p_{b, 2}$ is varied, where $p_{b, 1}$ is

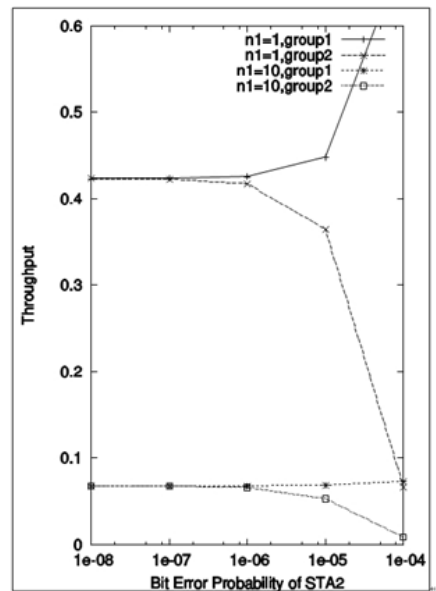

(a) $p_{b, 1}=10^{-8}$

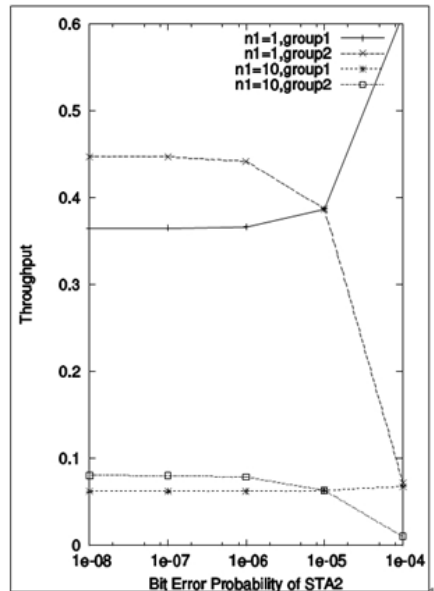

(b) $p_{b, 1}=10^{-5}$

Fig. 3. Station throughput versus $p_{b, 2}$ for $n_{1}=1,10$ and $n_{2}=1$

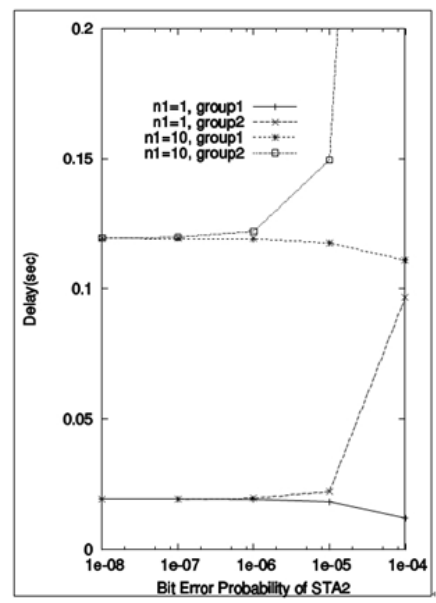

(a) $p_{b, 1}=10^{-8}$

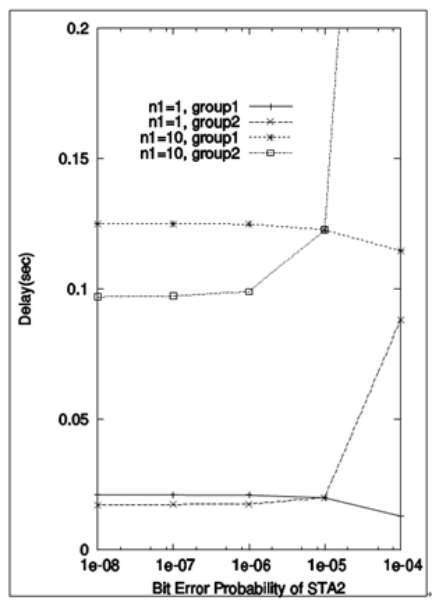

(b) $p_{b, 1}=10^{-5}$

Fig. 4. Packet delay versus $p_{b, 2}$ for $n_{1}=1,10$ and $n_{2}=1$ 
Table 2. The percentage of the throughput variations when $\mathrm{p}_{\mathrm{b}, 2}=10^{-5}$ compared with the throughput when $p_{b, 2}=10^{-8}$, where $p_{b, 1}$ is fixed at $10^{-8}$

\begin{tabular}{c|c|c|c|c}
\hline \multirow{2}{*}{$p_{b, 2}$} & \multicolumn{2}{|c|}{$n_{1}=1, n_{2}=1$} & \multicolumn{2}{c}{$n_{1}=10, n_{2}=1$} \\
\cline { 2 - 5 } & STA1 & STA2 & STA1 & STA2 \\
\hline $10^{-8}$ & 0.423262 & 0.423262 & 0.067700 & 0.067700 \\
\hline $10^{-5}$ & 0.448079 & 0.364723 & 0.069586 & 0.053028 \\
\hline$\%$ & +5.863 & -13.830 & +2.786 & -21.672 \\
\hline
\end{tabular}

Table 3. Throughput degradations of STA2 versus the number of stations in group 1 , where $\mathrm{p}_{\mathrm{b}, 1}$ is fixed at $10^{-8}$ and $\mathrm{m}^{\prime}=6$

\begin{tabular}{c|c|c|c|c|c}
\hline \multicolumn{2}{c|}{$p_{b, 2}$} & $n_{1}=1$ & $n_{1}=10$ & $n_{1}=20$ & $n_{1}=30$ \\
\hline \multirow{4}{*}{$m=5$} & $10^{-8}$ & 0.42326 & 0.06770 & 0.03249 & 0.02059 \\
\cline { 2 - 6 } & $10^{-5}$ & 0.36472 & 0.05302 & 0.02552 & 0.01653 \\
\cline { 2 - 6 } & $\%$ & -13.830 & -21.672 & -21.453 & -19.718 \\
\hline \multirow{3}{*}{$m=9$} & $10^{-8}$ & 0.42326 & 0.06791 & 0.03312 & 0.02127 \\
\cline { 2 - 6 } & $10^{-5}$ & 0.36465 & 0.05097 & 0.02396 & 0.01551 \\
\cline { 2 - 6 } & $\%$ & -13.847 & -24.944 & -27.657 & -27.080 \\
\hline
\end{tabular}

fixed at $10^{-8}$ in Fig. 3 (a) (or Fig. 4 (a)) and at $10^{-5}$ in Fig. 3 (b) (or Fig. 4 (b)). That is, we consider the impact of $p_{b, 2}$ on the performance for the following two cases: (i) group 1 is good, or (ii) there are bad conditions. Fig. 3 (a) illustrates that when $p_{b, 2}$ increases, the throughput of STA2 degrades; however the throughput of STA1 increases. This is because the IEEE 802.11 DCF governs the performance anomaly so called "the gap between the rich and the poor is getting bigger”. That is, if one station suffers from high FER, then its back-off stage should be increased so that the others get the benefit of smaller collision probabilities. This impact is then recursively applied to make the performance of the victim station worse. On the contrary, Fig. 3 (b) shows a totally different situation compared with Fig. 3 (a) that illustrates the impact of better FER of STA2 on the performance of both groups.

An interesting result is that the throughput of STA2 greatly varies in both cases. The throughput of STA2 for the first case sharply decreases by $21.7 \%$ (or $13.8 \%$ ) at the BER threshold $10^{-5}$ when $n_{1}=10$ (or $n_{1}=1$ )(see Table 2). On the contrary, the throughput of STA2 for the second case increases by $27.1 \%$ (or $15.5 \%$ ) when $p_{b, 2}=10^{-8}$ and $n_{1}=10$ (or $n_{1}=1$ ). Therefore we can easily expect that the percent of the throughput variations of STA2 depends on the number of group 1 as well as $p_{b, 1}$ and $p_{b, 2}$. However, Table 3 shows that the percent of the performance degradations of STA 2 might be saturated when $n_{1}=20$. If we increase the retry limit number of the DCF parame-

Table 4. The percentage of the average packet delay variations when $p_{b, 2}=10^{-5}$ compared with the throughput when $\mathrm{p}_{\mathrm{b}, 2}=10^{-8}$, where $\mathrm{p}_{\mathrm{b}, 1}$ is fixed at $10^{-8}$

\begin{tabular}{c|c|c|c|c}
\hline \multirow{2}{*}{$p_{b, 2}$} & \multicolumn{2}{|c|}{$n_{1}=1, n_{2}=1$} & \multicolumn{2}{c}{$n_{1}=10, n_{2}=1$} \\
\cline { 2 - 5 } & STA1 & STA2 & STA1 & STA2 \\
\hline $10^{-8}$ & 0.019333 & 0.019333 & 0.119376 & 0.119376 \\
\hline $10^{-5}$ & 0.018281 & 0.022347 & 0.117625 & 0.149557 \\
\hline$\%$ & -4.441 & +15.899 & -1.466 & +25.282 \\
\hline
\end{tabular}


ter, the throughput degradation of STA2 should be deeper as thenum ber of stations in class 1 increases (see Table 3).

Fig. 4 and Table 4 show the performance of average packet delay for our second numerical scenario. As explained earlier in the throughput performance, the delay performance is also considerably worse as compared to the first numerical scenario.

\section{Simulation Study}

In this section, we compare the analytic results in the previous section with simulation using the OPNET [10]. For the simulation, we created a scenario composed of a single BSS connected to a wire-line distributed network with a fixed AP, an Ethernet switch, two servers, and two groups of stations (see Fig. 5). We modeled the wireless/wireline network system as a dumbbell network where $n_{1}$ stations in group 1 access server 1 and a station (STA2) in group 2 accesses server2 through the AP. The stations in group 1 are fixed, and STA2 follows a pre-defined trajectory of 112 meters. We simulate 240 seconds during which the STA2 proceeds to the AP, stops at 32 and 75 meters, and arrives at the end of the trajectory. The STA2 stays at each stop for 600 seconds. Each station generates a single UDP stream of data frames that are 1,023 bytes long at a high data rate in such a way that the WLAN is overloaded. We use the BERs of the frame transmission using a table look-up of the DPSK by the OPNET (see Table 5). We also use the same station parameters shown in Table 1.

Fig. 6(a) plots the system throughput of each group and the received and forward traffic of the AP, where $n_{1}=1,10$. To evaluate the accuracy of the analytic models, we provide the system

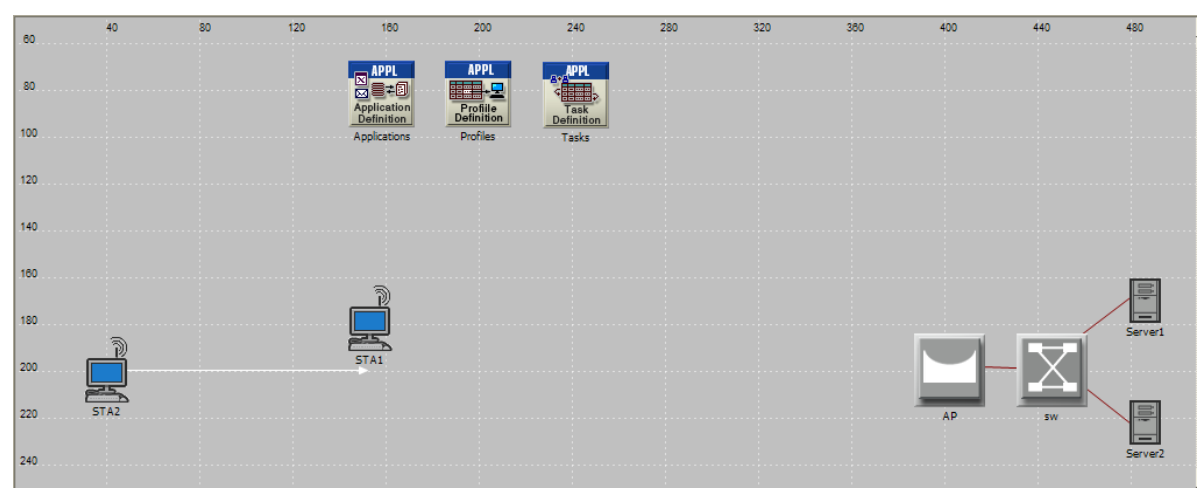

Fig. 5. The OPNET simulation scenario for the single BSS

Table 5. Analytic system throughputs for the simulation intervals

\begin{tabular}{c|c|c|c|c|c}
\hline \multirow{2}{*}{$\begin{array}{c}\text { Simulation } \\
\text { Time }(\mathrm{sec})\end{array}$} & \multirow{2}{*}{$\begin{array}{c}P_{b, 2} \\
\text { (OPNET) }\end{array}$} & STA1 & STA2 & \multicolumn{2}{c}{$n_{1}=1, n_{2}=1$} \\
\cline { 3 - 5 } & 0.0001220 & 0.704 & 0.047 & 0.732 & 0.0064 \\
\hline $0-600$ & 0.0000226 & 0.484 & 0.297 & 0.699 & 0.0390 \\
\hline $601-1200$ & 0.0000009 & 0.425 & 0.417 & 0.678 & 0.0659 \\
\hline $1201-1800$ & $10^{-8}$ & 0.423 & 0.423 & 0.676 & 0.0676 \\
\hline $1801-2400$ & &
\end{tabular}




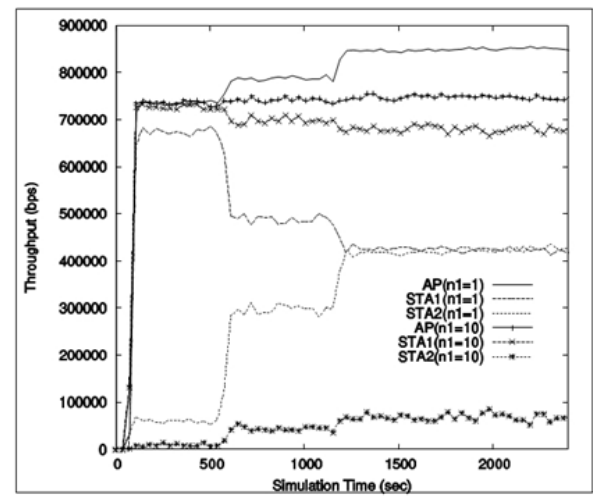

(a) System Throughput

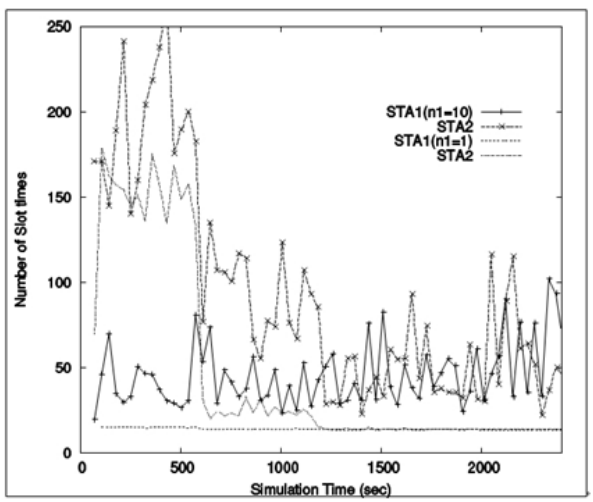

(b) Average Number of Sot Times

Fig. 6. Simulation results for two groups of stations, where $n_{2}=1$ and $n_{1}=1,10$

Table 6. Average number of slot times for successful packet transmissions computed by the analytic model for the simulation intervals

\begin{tabular}{c|c|c|c|c|c}
\hline \multirow{2}{*}{$\begin{array}{c}\text { Simulation } \\
\text { Time (sec) }\end{array}$} & \multirow{2}{*}{$\begin{array}{c}P_{b, 2} \\
(\text { OPNET) }\end{array}$} & STA1 & STA2 & STA1 & STA2 \\
\cline { 3 - 5 } & 0.0001220 & 15.86 & 177.17 & 36.34 & 247.66 \\
\hline $0-600$ & 0.0000226 & 16.97 & 28.06 & 37.32 & 64.536 \\
\hline $601-1200$ & 0.0000009 & 17.47 & 17.85 & 38.00 & 39.107 \\
\hline $1201-1800$ & $10^{-8}$ & 17.49 & 17.54 & 38.05 & 38.050 \\
\hline $1801-2400$ & \multicolumn{2}{|c|}{} &
\end{tabular}

throughputs by the analytic results shown in Table 5 . These results closely follow the simulation results. Fig. 6 (b) plots the average number of slot times for the successful packet transmissions by the two stations of STA1 and STA2 in the simulation scenario. In order not to consider the propagation delay in detail between stations and the AP during simulations, we just plot the average number of slot times instead of the average packet delay. We note that we use an OPNET built-in statistic, which collects the number of slots a station needs to back-off before transmission while contenting for the medium, and the number of slots in the contention window after a station's successful transmission [10].

Table 6 shows the analytic results computed by equation (5) to compare with the simulation results shown in Fig. 6 (b). When $n_{1}=1$, the numerical results closely follow the simulation results. However, when $n_{1}=10$, it is not easy to compare them since the graphs shown in Fig. 8 fluctuates very widely. The simulation results will be more widely fluctuated as the number of contenting stations is increased because a station transmits packets less frequently in $1 \mathrm{Mbps}$ FHSS WLAN and then the updates of the statistics within the averaging time intervals of the simulation become less frequent. However we figured out that the average of number of slot times for each time interval of 600 seconds are very close to the analytic results for $n_{1}=10$.

\section{CONCLUSION}

We derived equations to compute the saturated throughput and the average packet delay of the 
IEEE 802.11 DCF in different FER conditions. This analysis is validated by comparison with simulation results. When all of the stations experience the same FER, which satisfied the receiver input level sensitivity, the throughput dropped by a maximum of $8.5 \%$ and the packet delay increased by a maximum up to $9.2 \%$ in the system values of the FHSS PHY layer. However, in the different FER conditions, the throughput and delay performances of a station suffering higher FER than others dropped under its deserved level. That is, the throughput and packet delay performances of the victim host dropped by over $20 \%$ as the number of stations in better conditions or the retry limit number of the DCF parameter was increased. In particular, we show the performance is considerably degraded even if the frame error rate of the victim station satisfies the receiver input level sensitivity that has been specified in the IEEE 802.11 standard. Hence, we must pay a lot more attention to co-channel interference in case of different frame error rates.

\section{REFERENCES}

[1] B. Crow, I. Widjaja, J. Kim and P. Sakai,“IEEE 802.11 Wireless Local Area Networks,” IEEE Communications Magazine, Vol.35, No.9, 1997, pp.116-126.

[2] E. Borgia, M. Conti and E. Gregori,"IEEE 802.11b Ad Hoc Networks: Performance Measurement," Journal of Cluster Computing, Vol.8, 2005, pp.135-145.

[3] M. Heusse, F. Rousseau, G. Berger-Sabbatel and A. Duda, "Performance anomaly of 802.11b," Proceedings of the IEEE Infocom, 2003.

[4] J. Choi, J. Na, K. Park and C. Kim, "Adaptive optimization of the rate adaptation algorithm in multirate WLANs," Proceedings of the IEEE International Conference on Network Protocols, 2007.

[5] IEEE, Part 11: Wireless LAN Medium Access Control (MAC) and Physical Layer (PHY) Specifications, IEEE Std 802.11 - 2007 (Revision of IEEE STd 802.11 - 1999, June, 2007.

[6] G. Bianchi, "Performance analysis of IEEE 802.11 distributed coordination function,"IEEE Journal of Selected Areas in Communications, Vol.18, No.3, 2000, pp.535-547.

[7] D. Malone, K. Duffy and D. Leith, "Modeling the 802.11 distributed coordination function in nonsaturated heterogeneous conditions," IEEE/ACM Trans. On Networking,Vol.15, No.1 2007, pp.159172.

[8] Q. Ni, T. Li, T. Turletti and Y. Xiao, "Saturation throughput analysis of error-prone 802.11 wireless networks," Wiley Journal of Wireless Communications and Mobile Computing, 2005.

[9] P. Chatzimisios, A. Boucouvalas and V. Vitsas, "Packet delay analysis of IEEE 802.11 MAC protocol,” Electronics Letters, Vol.39, No.18, 2003.

[10] OPNET, OPNET Modeler, http://www.opnet.com

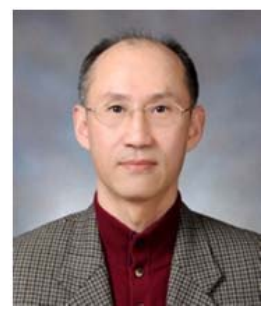

\section{Koohong Kang}

He received the BS and MS degrees in Electronics Engineering from Kyungpook National University and Chungnam National University, Korea, in 1985 and 1990, respectively, and then Ph.D. degree in Computer Science and Engineering from Pohang University Science and Technology (POSTECH), Korea, in 1998. During 1985-1993 and 1998-1999, he was with the Electronics and Telecommunications Research Institute (ETRI), Korea, as a member of technical staff. During 2008-2009, he stayed in the Dept. of Computer Science, Purdue University at West Lafayette, as a visiting scholor. He has been a professor in the Dept. of Information and Communication Engineering, Seowon University, Korea, since 2000. His research interests include wireless LANs, performance analysis and traffic control in high-speed network, and network security. 